\title{
Integrated analysis of IncRNA CTD-2357A8.3 expression and its potential roles in head and neck squamous cell carcinoma
}

\author{
BO ZOU ${ }^{1,2^{*}}$, XIN GUAN ${ }^{1,2^{*}}$, WEN ZHANG ${ }^{1,2}$, DONG WANG ${ }^{2,3}, \mathrm{KAI} \mathrm{XU}^{1,2}$, \\ DAO-YING YUAN ${ }^{2,3}, \mathrm{ZHEN} \mathrm{MENG}^{1-3}$ and BIN ZHANG ${ }^{1-3}$ \\ ${ }^{1}$ Department of Oral and Maxillofacial Surgery, Liaocheng People's Hospital, Medical College of Liaocheng University; \\ ${ }^{2}$ Key Laboratory of Oral Maxillofacial-Head and Neck Medical Biology of Shandong Province; \\ ${ }^{3}$ Precision Biomedical Key Laboratory, Liaocheng People's Hospital, Liaocheng, Shandong 252000, P.R. China
}

Received January 21, 2019; Accepted August 14, 2019

DOI: $10.3892 / \mathrm{ol} .2019 .10920$

\begin{abstract}
Head and neck squamous cell carcinoma (HNSCC), one of the most common malignant tumors, endangers human health. Recently, the incidence of HNSCC has kept increasing: However, its prognosis has not significantly improved. Understanding the molecular mechanism underlying HNSCC development will therefore provide new strategies for therapy. The present study attempted to identify differentially expressed (DE) long non-coding (lnc)RNAs and investigated their functional role in HNSCC development. Expression profiles of HNSCC and normal samples were downloaded from The Cancer Genome Atlas (TCGA) database. DElncRNAs between the HNSCC and normal samples were highlighted and their potential functions were investigated through lncRNA-micro (mi)RNA-mRNA network by using Gene Expression Profiling Interactive Analysis, UALCAN, DIANA-LncBase v.2 and miRWalk 3.0 databases. A total of 343 dysregulated lncRNAs were identified. Among these DElncRNAs, CTD-2357A8.3 had the highest fold-change and was significantly associated with poor overall survival in patients with HNSCC. Furthermore, CTD-2357A8.3 was associated with 'signaling pathways regulating stem cell pluripotency', 'proteoglycans in cancer', 'transcriptional
\end{abstract}

Correspondence to: Professor Bin Zhang, Department of Oral and Maxillofacial Surgery, Liaocheng People's Hospital, Medical College of Liaocheng University, 67 Dongchangxi Road, Liaocheng, Shandong 252000, P.R. China

E-mail: zbliaocheng@163.com

Dr Zhen Meng, Precision Biomedical Key Laboratory, Liaocheng People's Hospital, 67 Dongchangxi Road, Liaocheng, Shandong 252000, P.R. China

E-mail: chinamengzhen@163.com

"Contributed equally

Key words: head and neck squamous cell carcinoma, long non-coding RNA, CTD-2357A8.3, bioinformatical analysis, signaling pathway misregulation in cancer' and 'chemokine signaling pathway'. Further analysis demonstrated that CTD-2357A8.3 acted as a 'sponge' in order to competitively adsorb miRNA to regulate the expression of target gene caveolin 1 (CAVI) in HNSCC. In conclusion, CTD-2357A8.3 may be considered a promising diagnosis biomarker or a therapeutic target for the treatment of HNSCC.

\section{Introduction}

Head and neck squamous cell carcinoma (HNSCC) is the sixth most common type of human malignancy in United States $(1,2)$ and represents $\sim 4 \%$ of all new cancer cases in United States $(1,2)$. According to the latest statistics, $>700,000$ patients are affected every year worlwide, there are 358,000 cases of HNSCC-associated mortality cases and the incidence of HNSCC has significantly increased (3). Progress in clinical therapy technique has not significantly improved the survival rates of patients with HNSCC, and the 5-year survival rate of patients with $\mathrm{HNSCC}$ is only $40-50 \%$. Investigating the underlying mechanism of HNSCC development may offer novel strategies for its prevention, diagnosis and treatment through the development of potential biomarkers or therapeutic targets (4).

Long non-coding RNAs (lncRNAs) are defined as transcripts of over 200 nucleotides in length that possess no protein-coding function $(5,6)$. The human genome encodes tens of thousands of IncRNAs, which are associated with numerous physiological processes, including the regulation of transcription, translation, localization, and function of proteins (6). LncRNAs exert regulatory roles through different mechanisms. They can affect chromatin remodeling and methylation and be used as a functional sponge to competitively inhibit micro (mi)RNA and regulate the stability of protein complexes (7). It has been demonstrated that the abnormal expression of lncRNAs is associated with the occurrence of various types of human disease, including cancer (8). Furthermore, lncRNA has been reported to be involved in the regulation of HNSCC cell proliferation, differentiation and metastasis and to be associated with drug resistance $(9,10)$. However, the role of IncRNA in the development of HNSCC remains unclear. In particular, strong 
molecular markers that could be used to predict HNSCC prognosis are still missing.

The present study aimed therefore to identify differentially expressed (DE)lncRNAs between HNSCC and normal tissues via The Cancer Genome Atlas (TCGA) database. In addition, further analysis of TCGA will be performed to explore the prognostic value of these DElncRNAs. Eventually, the potential function and molecular mechanism of these DElncRNAs will be investigated through the IncRNA-miRNA-mRNA network.

\section{Materials and methods}

Data download and identification of DElncRNAs. The RNA sequence data (RNAseq) of HNSCC tissues and corresponding normal tissues were downloaded from TCGA database (https://www.cancer.gov/tcga). A total of 544 samples from 500 patients with HNSCC and 44 normal controls were collected in December 2018. The normal controls included normal tissues from the oral cavity, oral tongue, larynx, floor of the mouth and base of the tongue. The raw data were downloaded and limma package (version 3.2.5; https://www.r-project.org/) was used to screen DElncRNAs between HNSCC and normal tissues. A $\mid \log \mathrm{FCl}>2$ and adjusted $\mathrm{P}$-value $<0.05$ were set as the cut-off criteria.

Kaplan-Meier survival analysis. In order to determine whether the screened DElncRNAs possessed prognostic value, Kaplan-Meier survival analysis and log-rank test were performed by using Gene Expression Profiling Interactive Analysis (GEPIA) database (http://gepia.cancer-pku.cn/) (11). In GEPIA, the 'Methods' and 'Group Cutoff' were set as 'overall survival' and 'Median', respectively. Regarding mRNAs, their prognostic value was also verified by conducting log-rank analysis in UALCAN database (http://ualcan.path. uab.edu/analysis.html) (12).

Construction of IncRNA-miRNA-mRNA regulatory network. The DIANA-LncBase v.2 (DIANA Tools) $(13,14)$ was used to predict the target miRNAs of DElncRNA, and a score $>0.95$ was set as the screening threshold. The miRWalk 3.0 database (http://mirwalk.umm.uni-heidelberg.de/) was used to predict and screen the target mRNAs of the aforementioned miRNAs (15), and the filters were set as ' 0.95 ', '3UTR'and 'miRTarBase'. The Cytoscape software (version, 3.6.0; https://cytoscape.org/) (16) was used to visualize all the predicted results.

Gene Ontology $(G O)$ and Kyoto Encyclopedia of Genes and Genomes (KEGG) pathway enrichment analysis of target mRNAs. GO analysis (http://geneontology.org/) and KEGG pathway (https://www.genome.jp/) enrichment of differentially expressed genes (DEGs) were performed by using the Database for Annotation, Visualization, and Integrated Discovery (DAVID; version 6.7; https://david-d.ncifcrf.gov/) to screen the potential biological processes and signaling pathways in which the mRNAs are involved (17). The resulting data were imported into Cytoscape software to conduct visual analysis. $\mathrm{P}<0.05$ was considered to indicate a statistically significant difference.

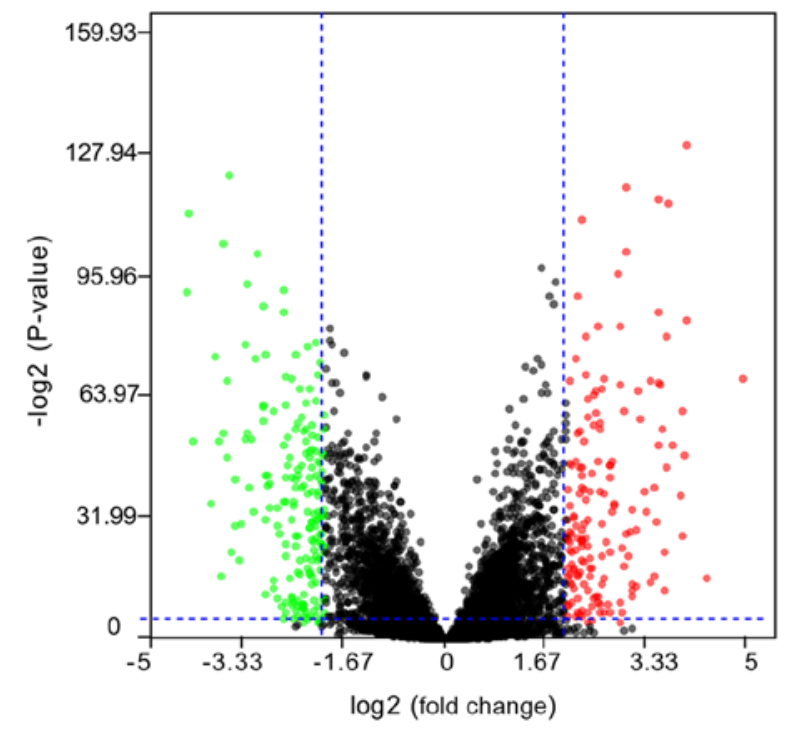

Figure 1. Differential expression of lncRNAs between HNSCC and normal tissues. Red points represent upregulated genes screened on the basis of $\mid \log \mathrm{FCl}>2.0$ and a corrected P-value $<0.05$. Green points represent downregulated genes screened on the basis of $\mid \log \mathrm{FCl}>2.0$ and a corrected P-value $<0.05$. Black points represent genes with no significant difference. FC, fold change.

Protein-protein interaction (PPI) network construction and hub genes identification. PPI networks were built by using the Search Tool for the Retrieval of Interacting Genes (STRING) software (version 11.0; http://string-db.org). The PPI network was visualized using Cytoscape software (18). By considering the node degree, Cytoscape CentiScaPe app (version, 3.6.0; http://apps.cytoscape.org/apps/centiscape) was used to screen hub genes in the network (19). Genes with a node degree $>5$ were considered to be candidate key genes.

Gene expression analysis. GEPIA was used to analyze the expression of DElncRNAs and mRNAs in HNSCC and normal tissues. Furthermore, the correlation between lncRNAs and mRNAs expression was analyzed using Pearson correlation in GEPIA (11). $\mathrm{P}<0.05$ was considered to indicate a statistically significant difference.

\section{Results}

Identification of DElncRNAs in HNSCC tissues. In the present study, RNAseq of HNSCC and corresponding normal tissue samples were downloaded from TCGA database. Following data screening, a total of 343 dysregulated IncRNAs were obtained, including 162 upregulated and 181 downregulated IncRNAs (Fig. 1).

Prognostic values of DElncRNAs. The association between the 343 DElncRNAs and the overall survival (OS) rate of patients with HNSCC was analyzed using GEPIA. The results demonstrated that the abnormal expression of 46 DElncRNAs was associated with the OS rate of patients with HNSCC $(\mathrm{P}<0.05)$. Furthermore, by applying more rigorous criteria $(\mathrm{P}<0.01)$, only 12 DElncRNAs were significantly associated with the OS rate of patients with HNSCC (Table I). CTD-2357A8.3, which was an IncRNA with the largest upregulation multiple, was 
Table I. Information of 12 frequently dysregulated long non-coding RNAs in head and neck squamous cell carcinoma identified by The Cancer Genome Atlas.

\begin{tabular}{llcccc}
\hline Ensembl ID & Gene symbol & Fold change & P-value & Up or down regulation & Log-rank P-value \\
\hline ENSG00000267123 & CTD-2357A8.3 & 3.96 & $3.43 \times 10^{-26}$ & Upregulated & 0.0087 \\
ENSG00000250874 & CTC-480C2.1 & 3.62 & $1.25 \times 10^{-4}$ & Upregulated & 0.001 \\
ENSG00000275216 & RP11-54H7.4 & 3.46 & $8.75 \times 10^{-13}$ & Upregulated & 0.0023 \\
ENSG00000233532 & LINC00460 & 3.31 & $6.37 \times 10^{-11}$ & Upregulated & 0.0019 \\
ENSG00000231131 & LNCAROD & 2.98 & $3.56 \times 10^{-09}$ & Upregulated & 0.0061 \\
ENSG00000277268 & LHX1-DT & 2.96 & $1.18 \times 10^{-06}$ & Upregulated & 0.0028 \\
ENSG00000259692 & RP11-499F3.2 & 2.36 & $2.63 \times 10^{-10}$ & Upregulated & 0.0029 \\
ENSG00000251185 & RP11-542G1.1 & 2.03 & $8.57 \times 10^{-08}$ & Upregulated & 0.0063 \\
ENSG00000215386 & MIR99AHG & -2.09 & $1.57 \times 10^{-13}$ & Downregulated & 0.00065 \\
ENSG00000176728 & TTTY14 & -2.48 & $6.02 \times 10^{-09}$ & Downregulated & 0.0037 \\
ENSG00000267709 & AC024592.9 & -2.60 & $7.76 \times 10^{-17}$ & Downregulated & 0.0068 \\
ENSG00000228789 & HCG22 & -4.29 & $1.99 \times 10^{-28}$ & Downregulated & 0.00065 \\
\hline
\end{tabular}

Table II. miRNAs targeting CTD-2357A8.3 predicted by LncBase Predicted v.2.

\begin{tabular}{lll}
\hline miRNAs & \multicolumn{1}{c}{ MirBase ID } & Score \\
\hline hsa-miR-7113-5p & MIMAT0028123 & 0.984 \\
hsa-miR-6895-3p & MIMAT0027691 & 0.984 \\
hsa-miR-3657 & MIMAT0018077 & 0.965 \\
hsa-miR-7856-5p & MIMAT0030431 & 0.964 \\
hsa-miR-346 & MIMAT0000773 & 0.96 \\
hsa-miR-3119 & MIMAT0014981 & 0.955 \\
hsa-miR-4514 & MIMAT0019051 & 0.955 \\
\hline
\end{tabular}

miRNAs, microRNAs.

selected for further analysis. GEPIA analysis demonstrated that CTD-2357A8.3 expression in HNSCC tissues was significantly increased compared to adjacent normal tissues, and was associated with OS rate of patients with $\operatorname{HNSCC}$ (Fig. 2).

LncRNA-miRNA-mRNA regulatory network. One of the important regulatory mechanisms of lncRNA is its competitive adsorption of miRNA as a 'sponge', which can be used to regulate the expression of target genes. The LncBase v.2 from DIANA Tools was used to predict the target miRNAs of DElncRNAs. For CTD-2357A8.3, hsa-miR-7113-5p, hsa-miR-6895-3p, hsa-miR-3657, hsa-miR-7856-5p, hsa-miR-346, hsa-miR-3119 and hsa-miR-4514 were identified as candidate target miRNAs (Table II). In addition, miRWalk 3.0 was used to predict and screen the target mRNAs of these miRNAs. These mRNAs were eventually validated by using miRTarBase. A total of 213 mRNAs were identified as the target genes of the aforementioned miRNAs and were visualized by using Cytoscape (Fig. 3).

GO analysis and KEGG pathway enrichment of target genes. GO and KEGG pathway analysis of the 213 mRNAs were performed using DAVID database. The criterion was $\mathrm{P}<0.05$. As presented in Fig. 4, the results from KEGG pathway enrichment demonstrated that mRNAs were associated with 'signaling pathways regulating pluripotency of stem cells' (hsa04550, $\mathrm{P}=0.003$ ), 'proteoglycans in cancer' (hsa05205, $\mathrm{P}=0.02$ ), 'transcriptional misregulation in cancer' (hsa05202, $\mathrm{P}=0.033$ ) and 'chemokine signaling pathway' (hsa04062, $\mathrm{P}=0.049$ ). In addition, the GO terms of biological functions were mainly associated with 'regulation of transcription' (GO:0006355, $\mathrm{P}=0.014)$, 'negative regulation of cell proliferation' (GO:0008285, $\mathrm{P}=0.006)$ and 'angiogenesis' (GO:0001525, $\mathrm{P}=0.001)$.

Caveolin 1 (CAVI) was identified as the target gene of CTD-2357A8.3. The PPI network of the candidate target mRNAs was constructed using STRING database and visualized by Cytoscape software. In total, 110 mRNAs were entered into the PPI network complex (Fig. 5). With node degree $>5$ as inclusion criteria, the Cytoscape CentiScaPe was used to screen candidate key genes in the network. A total of 13 genes met the criteria and will be further investigated (Table III). Pearson's correlation between CTD-2357A8.3 and these 13 genes was analyzed using GEPIA, and the results demonstrated that the expression of glycogen synthase kinase $3 \beta$ (GSK3B), CAV1, Rho GDP dissociation inhibitor $\alpha$ (ARHGDIA) and cyclin dependent kinase 9 (CDK9) was positively correlated with CTD-2357A8.3 (Table III and Fig. 6). In addition, GEPIA and UALCAN were used to assess the association between these hub genes and OS of patients with HNSCC. The results demonstrated that only CAV1 was significantly associated with OS in the two databases, and CAV1 expression in HNSCC tissues was significantly higher than that in normal tissues (Table III and Fig. 6). Therefore, according to 'ceRNA Hypothesis', messenger RNAs, transcribed pseudogenes and lncRNAs can 'talk' to each other using microRNA response elements as letters of a new language (20). So, CAV1 may therefore be considered as a potential target of CTD-2357A8.3 in patients with HNSCC. 

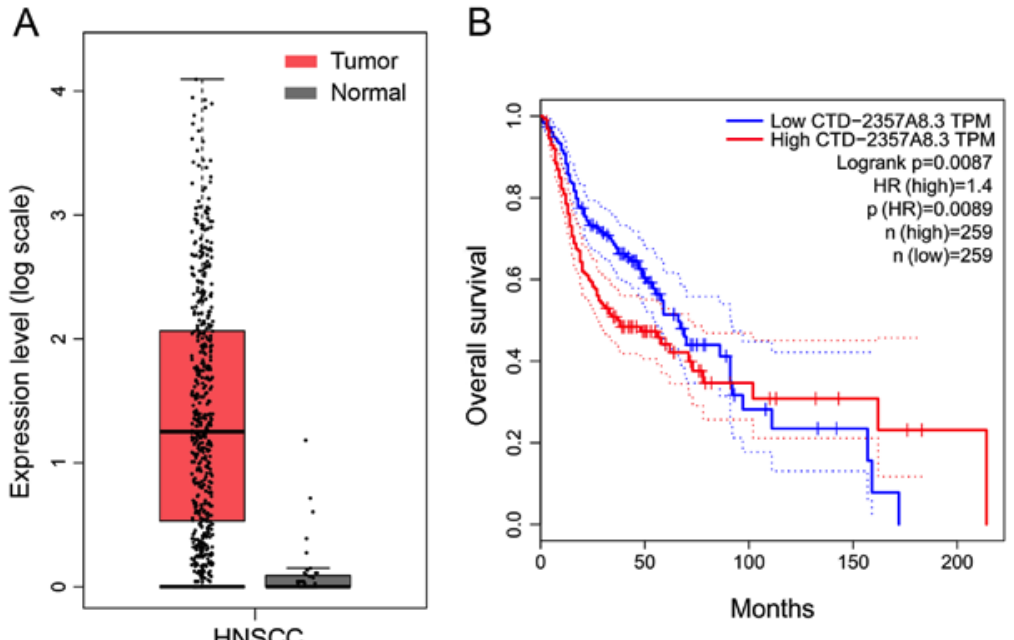

Figure 2. Expression level of CTD-2357A8.3 in HNSCC and its association with the OS of patients with HNSCC. (A) CTD-2357A8.3 was highly expressed in HNSCC tissues compared with normal tissues. Red represents tumor tissues and grey represents normal tissues. (B) Log-rank analysis demonstrated that the OS of patients with HNSCC and with high CTD-2357A8.3 expression was significantly reduced $(\mathrm{P}<0.05)$. HR, hazard ratio; OS, overall survival.

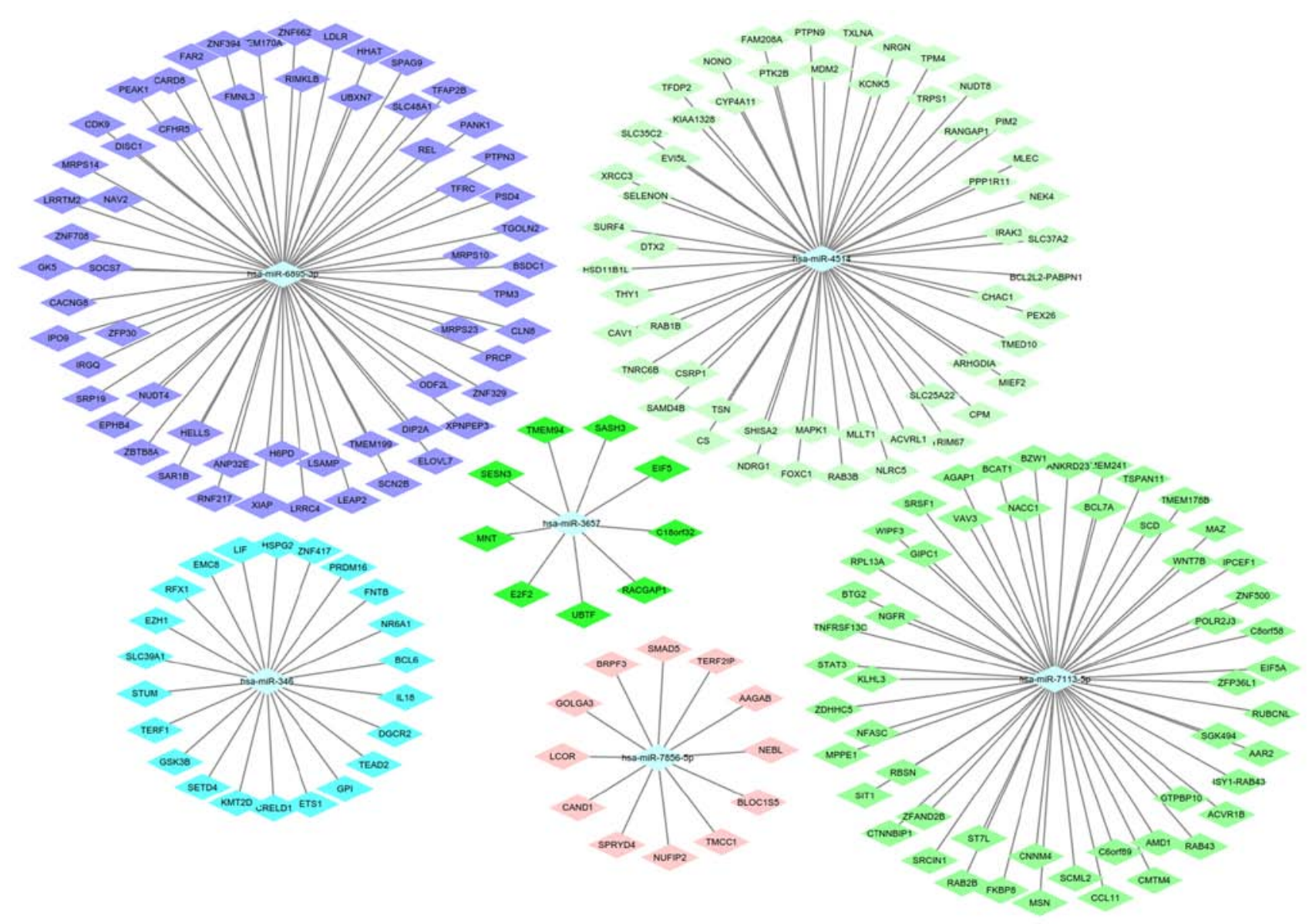

Figure 3. Construction of long non-coding RNA-miRNA-mRNA regulatory network. hsa-miR-7113-5p, hsa-miR-6895-3p, hsa-miR-3657, hsa-miR-7856-5p, hsa-miR-346, hsa-miR-3119 and hsa-miR-4514 were identified as the candidate target miRNAs. The target mRNAs of miRNAs were predicted using miRWalk3.0 and validated by at least one method using miRTarBase. miRNAs, mircoRNAs.

\section{Discussion}

HNSCC is one of the most common types of human malignancy. HNSCC is characterized by a rapid progression, high migratory capacity and high mortality rate; however, almost no biomarkers or targets exist for the diagnosis and therapy of HNSCC (2). The determination of DEGs between tumor and normal tissues may therefore help exploring the pathogenesis of HNSCC and providing potential novel biomarkers or targets for early diagnosis and therapy of HSNCC. 


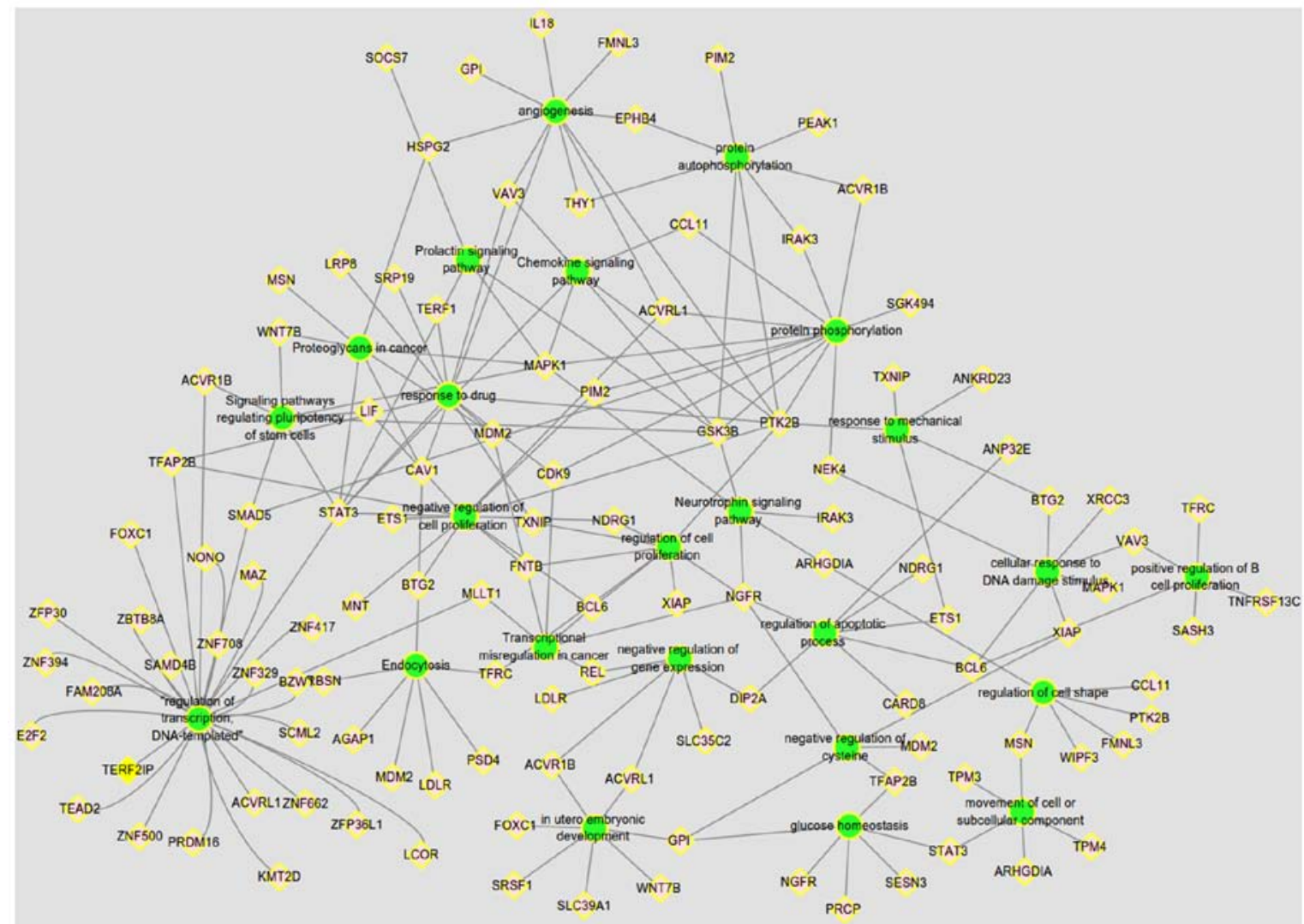

Figure 4. Gene Ontology and Kyoto Encyclopedia of Genes and Genomes pathway analysis of the target genes of CTD-2357A8.3 in HNSCC tissues. Analysis of the 213 mRNAs was performed using the Database for Annotation, Visualization, and Integrated Discovery and visualized using Cytoscape. 'Signaling pathways regulating pluripotency of stem cells' (hsa04550, $\mathrm{P}=0.003$ ), 'proteoglycans in cancer' (hsa05205, $\mathrm{P}=0.02$ ), 'transcriptional misregulation in cancer' (hsa05202, $\mathrm{P}=0.033$ ) and 'chemokine signaling pathway' (hsa04062, $\mathrm{P}=0.049$ ) were the top four significantly enriched gene sets.

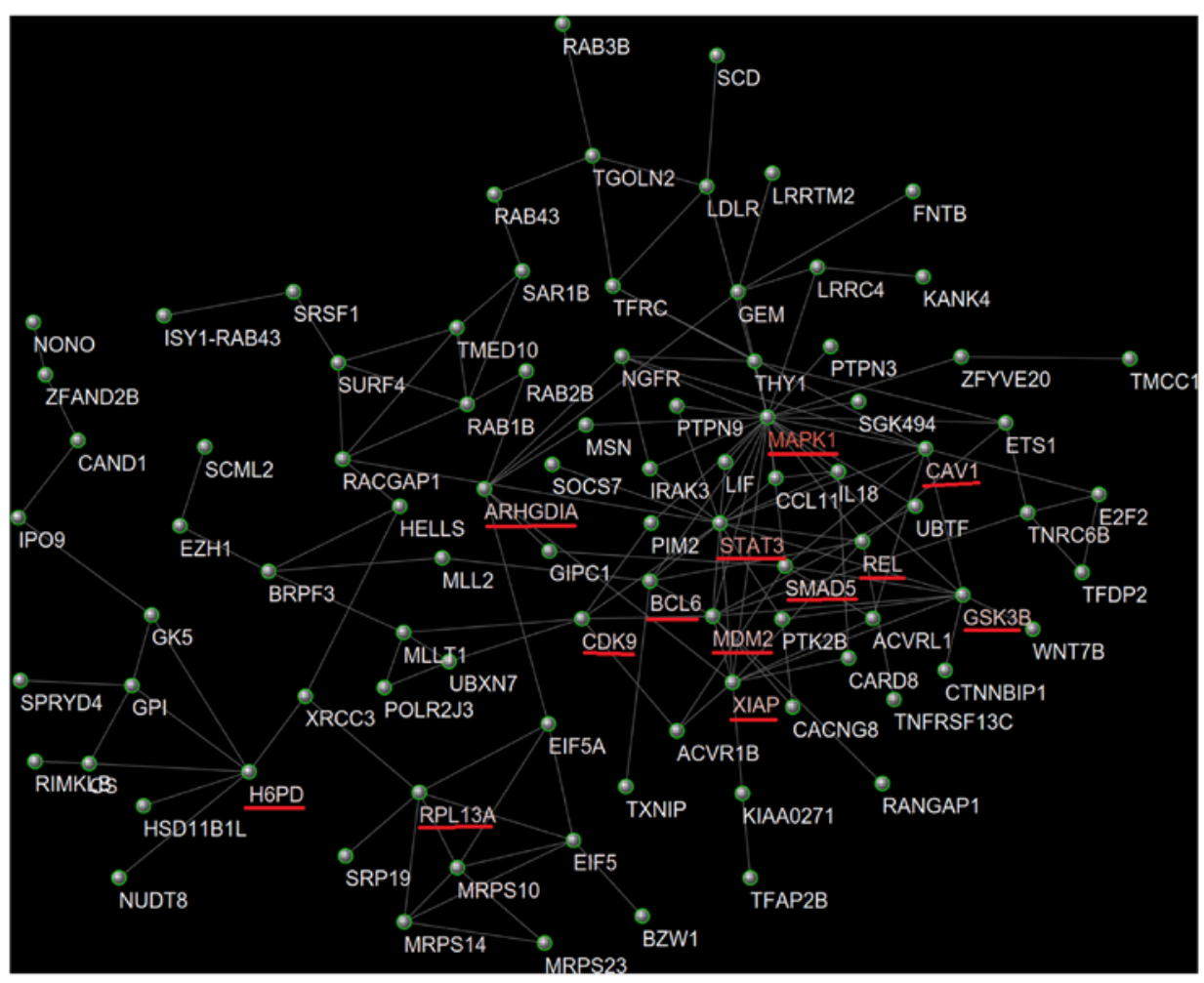

Figure 5. Identification of hub genes. The PPI network of the candidate target mRNAs was constructed using the Search Tool for the Retrieval of Interacting Genes software and visualized by Cytoscape. Confidence score $\geq 0.4$ was set as the cutoff criterion and total of 110 genes were filtered into the PPI network complex. The gene degree was further analyzed by CentiScaPe 2.2 plugin of Cytoscape. Genes with degree $>5$ were identified as hub genes. PPI, protein-protein interaction. 
Table III. Pearson correlation analysis between CTD-2357A8.3 and 12 hub genes expressions and the log-rank analysis of these genes.

\begin{tabular}{lcccc}
\hline mRNA & r (Pearson) & P-value (Pearson) & Log-rank P-value (GEPIA) & Log-rank P-value (UALCAN) \\
\hline MAPK1 & 0.029 & 0.49 & 0.54 & 0.8 \\
STAT3 & -0.13 & 0.002 & 0.1 & 0.12 \\
MDM2 & -0.11 & 0.0095 & 0.53 & 0.096 \\
XIAP & 0.075 & 0.076 & 0.53 & 0.66 \\
GSK3B & 0.14 & 0.0013 & 0.13 & 0.42 \\
CAV1 & 0.42 & 0 & 0.0016 & 0.038 \\
ARHGDIA & 0.37 & 0 & 0.0028 & 0.26 \\
H6PD & -0.021 & 0.61 & 0.44 & 0.038 \\
RPL13A & -0.12 & $5.2 \times 10-3$ & 0.9 & 0.2 \\
SMAD5 & -0.11 & 0.013 & 0.3 & 0.96 \\
CDK9 & 0.2 & $1.3 \times 10-06$ & 0.043 & 0.083 \\
BCL6 & -0.21 & $4.4 \times 10-07$ & 0.16 & 0.88 \\
REL & -0.015 & 0.73 & 0.45 & 0.18 \\
\hline
\end{tabular}

ARHGDIA, Rho GDP dissociation inhibitor $\alpha$; BCL6, BCL6 transcription repressor; CDK9, cyclin dependent kinase 9; GEPIA, Gene Expression Profiling Interactive Analysis; GSK3B, glycogen synthase kinase $3 \beta$; H6PD, hexose-6-phosphate dehydrogenase/glucose 1-dehydrogenase; MAPK1, mitogen-activated protein kinase 1; MDM2, MDM2 proto-oncogene; REL, REL proto-oncogene, NF-kB subunit; RPL13A, ribosomal protein L13a; SMAD5, SMAD family member 5; STAT3, signal transducer and activator of transcription 3; CAV1, caveolin 1; XIAP, X-linked inhibitor of apoptosis.
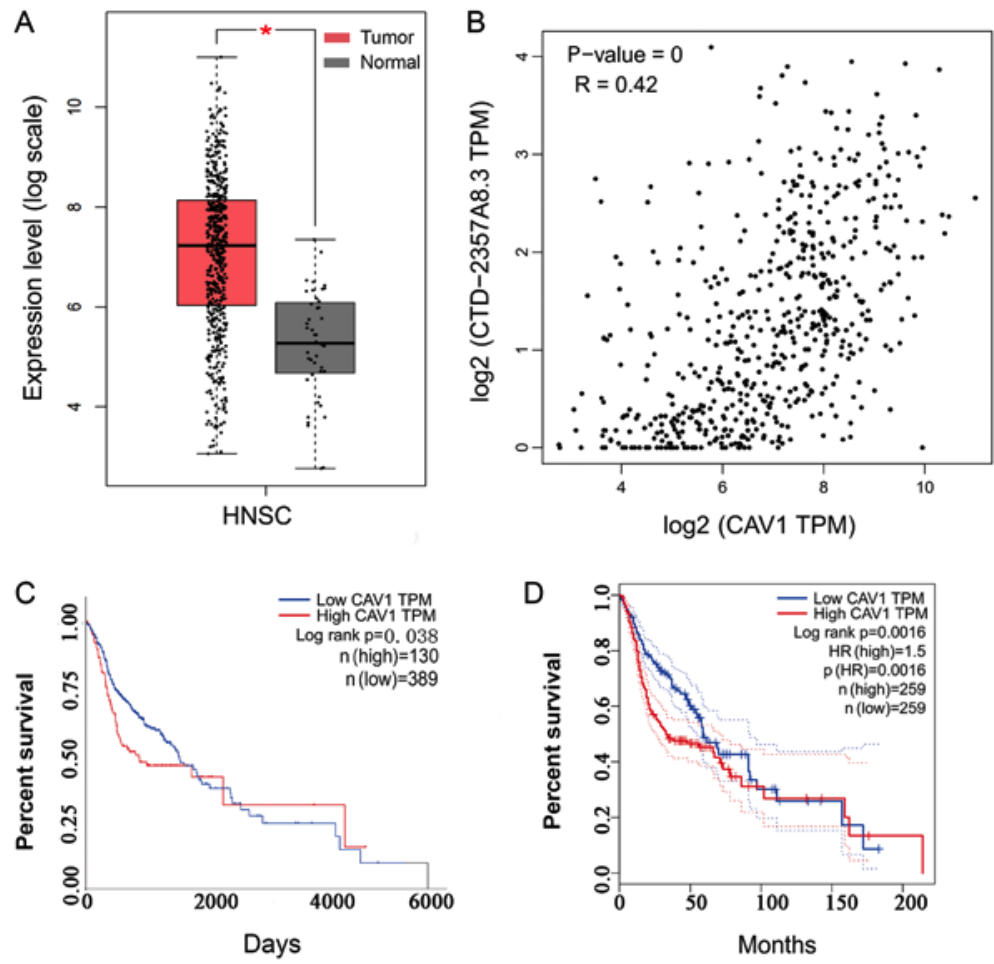

Figure 6. CAV1 was identified as the target gene of CTD-2357A8.3. (A) CAV1 expression level was increased in HNSCC tissue compared with normal tissues. Red represents tumor tissues and gray represents normal tissues. (B) Pearson correlation analysis of CAV1 and CTD-2357A8.3 expression in HSNCC tissues. (C) Kaplan-Meier survival analysis comparing the high or low CAV1 expression with overall survival of patients with HSNCC according to data from UALCAN database. (D) Kaplan-Meier survival analysis comparing the high or low CAV1 expression with overall survival of patients with HSNCC according to data from Gene Expression Profiling Interactive Analysis database. CAV1, caveolin 1; HSNCC, head and neck squamous cell carcinoma.

Recently, lncRNAs have attracted increasing interest. In cancer, some lncRNAs have been demonstrated to function as oncogenes, whereas others inhibit invasion and metastasis by participating in various cellular processes, including proliferation and differentiation (21). Some lncRNAs serve important role in the development and progression of HNSCC, and it 
has been reported that these lncRNAs could be used as novel biomarkers and monitoring tools and as potential therapeutic targets in HNSCC treatment (22). Exploring the lncRNA differences between HNSCC and normal tissues could therefore provide a better understanding of the mechanism involved in the occurrence and development of HNSCC, and offer a basis for the development of novel diagnostic markers and therapeutic targets of HNSCC.

In the present study, the abnormally expressed lncRNAs in HSNCC were explored using TCGA database, and 343 dysregulated lncRNAs were identified, of which 162 lncRNAs were upregulated and 181 were downregulated. Following log-rank survival analysis, 12 lncRNAs were found to be significantly associated with the OS of patients with HNSCC. Among these 1ncRNAs, CTD-2357A8.3 had the largest $\mid \log \mathrm{FCl}$ and was therefore further analyzed. The results demonstrated that CTD-2357A8.3 expression in HNSCC tissues was significantly increased compared to normal tissues and was associated with the OS rate of patients with HNSCC. However, following a detailed analysis of the literature, no relevant study on the role of CTD-2357A8.3 in HNSCC was found so far. Subsequently, it is really necessary and imperative to make further in-depth study investigating the specific role of CTD-2357A8.3 in HNSCC.

One of the important regulatory mechanisms of lncRNA is its competitive adsorption of miRNA as a 'sponge'. Subsequently, lncRNA serves a crucial role in regulating the expression of target genes. The candidate target miRNAs and mRNAs of CTD-2357A8.3 were therefore predicted in the present study. The GO and KEGG pathway analysis of the 213 target mRNAs reported that, 'signaling pathways regulating pluripotency of stem cells', 'proteoglycans in cancer', 'transcriptional misregulation in cancer' and 'chemokine signaling pathway' were the top significantly enriched gene sets and were all associated with cancer.

According to the mechanism of IncRNA, the expression of IncRNA and mRNA should be consistent. In the present study, the hub genes were identified by integrating centrality analysis with Pearson correlation and log-rank survival analysis. The results demonstrated that GSK3B, CAV1, ARHGDIA and CDK9 expression was positively correlated with CTD-2357A8.3. Furthermore, only CAV1 was significantly associated with the OS of patients with HNSCC according to GEPIA and UALCAN databases. These results suggested that CAV1 may be considered as a potential target gene for CTD-2357A8.3. CAV1 is a membrane-bound scaffold protein that can modulate signal transduction; however, the role of CAV1 in cancer is dependent on the tissue source (23). It has been reported that CAV1 inhibits cell migration and invasion by inhibiting epithelial mesenchymal transformation in pancreatic cancer (24). Furthermore, CAV1 has been reported to be highly expressed in colon cancer cells that have low metastatic ability, but not in colon cancer cells with high metastatic ability. These results suggest that CAV1 is involved in the negative regulation of tumor cell metastatic ability (25). Furthermore, in clear cell renal cell carcinoma and lung cancer, the increased expression of CAV1 was demonstrated to promote cell proliferation and invasion, and was positively correlated with poor prognosis $(26,27)$. Further investigation is therefore required to verify whether CTD-2357A8.3 could regulate CAV1 expression through miRNA, and to explore the functional roles of CTD-2357A8.3 in the tumorigenesis of HNSCC. In addition, the present study used the GEPIA database to analyze the association between CTD-2357A8.3, CAV1 and the OS in HNSCC. This database contains RNA sequencing expression data of HNSCC from different primary sites, including the tonsil, oropharynx, oral cavity, larynx and hypopharynx. Further investigation is therefore required to determine whether CTD-2357A8.3 from different primary sites would remain positively correlated with the OS of patients with HNSCC. In addition, it may be also or even more important to consider the disease-free survival in HNSCC analysis.

\section{Acknowledgements}

Not applicable.

\section{Funding}

This study was supported by the Medicine and Health Science Technology Development Plan Project, Shandong Province (grant no. 2017WSA15041), the National Natural Science Foundation of China (grant nos. 81472530 and 81602374) and the Natural Science Foundation of Shandong Province (grant no. 2016ZRA15065).

\section{Availability of data and materials}

All data generated or analyzed during the present study are included in this published article.

\section{Authors' contributions}

BoZ and XG downloaded the data, performed the identification of DElncRNAs and PPI network construction and drafted the manuscript. WZ and DW performed the survival analysis and constructed the IncRNA-miRNA-mRNA regulatory network. KX performed GO analysis and KEGG pathway enrichment analysis. DY was involved in the PPI network construction. $\mathrm{ZM}$ analyzed the association between the candidate genes and prognosis, and also participated the design of the study. BiZ was the major contributor in designing the present study. All authors read and approved the final version of the manuscript.

\section{Ethics approval and consent to participate}

Not applicable.

\section{Patient consent for publication}

Not applicable.

\section{Competing interests}

The authors declare that they have no competing interests.

\section{References}

1. Siegel RL, Miller KD and Jemal A: Cancer statistics, 2019. CA Cancer J Clin 69: 7-34, 2019. 
2. Siegel RL, Miller KD and Jemal A: Cancer statistics, 2018. CA Cancer J Clin 68: 7-30, 2018.

3. Bray F, Ferlay J, Soerjomataram I, Siegel RL, Torre LA and Jemal A: Global cancer statistics 2018: GLOBOCAN estimates of incidence and mortality worldwide for 36 cancers in 185 countries. CA Cancer J Clin 68: 394-424, 2018.

4. Goossens N, Nakagawa S, Sun X and Hoshida Y: Cancer biomarker discovery and validation. Transl Cancer Res 4: 256-269, 2015

5. Iyer MK, Niknafs YS, Malik R, Singhal U, Sahu A, Hosono Y, Barrette TR, Prensner JR, Evans JR, Zhao S, et al: The landscape of long noncoding RNAs in the human transcriptome. Nat Genet 47: 199-208, 2015.

6. Wilusz JE, Sunwoo H and Spector DL: Long noncoding RNAs: Functional surprises from the RNA world. Genes Dev 23 1494-1504, 2009.

7. Li T, Mo X, Fu L, Xiao B and Guo J: Molecular mechanisms of long noncoding RNAs on gastric cancer. Oncotarget 7: 8601-8612, 2016

8. Park S, Lee M, Chun CH and Jin EJ: The lncRNA, nespas, is associated with osteoarthritis progression and serves as a potential new prognostic biomarker. Cartilage 10: 148-156, 2019.

9. Wang R, Ma Z, Feng L, Yang Y, Tan C, Shi Q, Lian M, He S, Ma H and Fang J: LncRNA MIR31HG targets HIF1A and P21 to facilitate head and neck cancer cell proliferation and tumorigenesis by promoting cell-cycle progression. Mol Cancer 17: 162, 2018

10. Wang Y, Wu C, Zhang C, Li Z, Zhu T, Chen J, Ren Y, Wang X, Zhang L and Zhou X: TGF- $\beta$-induced STAT3 overexpression promotes human head and neck squamous cell carcinoma invasion and metastasis through malat1/miR-30a interactions. Cancer Lett 436: 52-62, 2018

11. Tang Z, Li C, Kang B, Gao G, Li C and Zhang Z: GEPIA: A web server for cancer and normal gene expression profiling and interactive analyses. Nucleic Acids Res 45 (W1): W98-W102, 2017.

12. Chandrashekar DS, Bashel B, Balasubramanya SAH, Creighton CJ, Ponce-Rodriguez I, Chakravarthi BVSK and Varambally S: UALCAN: A portal for facilitating tumor subgroup gene expression and survival analyses. Neoplasia 19: 649-658, 2017.

13. Vlachos IS and Hatzigeorgiou AG: Functional analysis of miRNAs using the DIANA tools online suite. Methods Mol Biol 1517: 25-50, 2017.

14. Paraskevopoulou MD, Vlachos IS and Hatzigeorgiou AG DIANA-TarBase and DIANA suite tools: Studying experimentally supported microRNA targets. Curr Protoc Bioinformatics 55: 12.14.1-12.14.18, 2016

15. Sticht C, De La Torre C, Parveen A and Gretz N: miRWalk: An online resource for prediction of microRNA binding sites. PLoS One 13: e0206239, 2018.

16. Kohl M, Wiese S and Warscheid B: Cytoscape: Software for visualization and analysis of biological networks. Methods Mol Biol 696: 291-303, 2011.
17. Huang da W, Sherman BT and Lempicki RA: Systematic and integrative analysis of large gene lists using DAVID bioinformatics resources. Nat Protoc 4: 44-57, 2009.

18. Franceschini A, Szklarczyk D, Frankild S, Kuhn M, Simonovic M, Roth A, Lin J, Minguez P, Bork P, von Mering C and Jensen LJ: STRING v9.1: Protein-protein interaction networks, with increased coverage and integration. Nucleic Acids Res 41 (Database Issue): D808-D815, 2013.

19. Scardoni G, Tosadori G, Faizan M, Spoto F, Fabbri F and Laudanna C: Biological network analysis with CentiScaPe: Centralities and experimental dataset integration. F1000Res 3: 139,2014

20. Salmena L, Poliseno L, Tay Y, Kats L and Pandolfi PP: A ceRNA hypothesis: The Rosetta Stone of a hidden RNA language? Cell 146: 353-358, 2011

21. Sun M and Kraus WL: From discovery to function: The expanding roles of long non-coding RNAs in physiology and disease. Endocr Rev: er00009999, 2015 (Epub ahead of print).

22. Song W, Sun Y, Lin J and Bi X: Current research on head and neck cancer-associated long noncoding RNAs. Oncotarget 9 1403-1425, 2017.

23. Parton RG and Simons K: The multiple faces of caveolae. Nat Rev Mol Cell Biol 8: 185-194, 2007.

24. Salem AF, Bonuccelli G, Bevilacqua G, Arafat H, Pestell RG, Sotgia F and Lisanti MP: Caveolin-1 promotes pancreatic cancer cell differentiation and restores membranous E-cadherin via suppression of the epithelial-mesenchymal transition. Cell Cycle 10: 3692-3700, 2011.

25. Nimri L, Barak H, Graeve L and Schwartz B: Restoration of caveolin-1 expression suppresses growth, membrane-type-4 metalloproteinase expression and metastasis-associated activities in colon cancer cells. Mol Carcinog 52: 859-870, 2013.

26. Joo HJ, Oh DK, Kim YS, Lee KB and Kim SJ: Increased expression of caveolin-1 and microvessel density correlates with metastasis and poor prognosis in clear cell renal cell carcinoma. BJU Int 93: 291-296, 2004.

27. Liu W, Yin NC, Liu H and Nan KJ: Cav-1 promote lung cancer cell proliferation and invasion through lncRNA HOTAIR. Gene 641: 335-340, 2018.

This work is licensed under a Creative Commons Attribution-NonCommercial-NoDerivatives 4.0 International (CC BY-NC-ND 4.0) License. 